\title{
Radio Search for EHE Neutrinos with the ARIANNA Pilot Array, and ARIANNA Performance Projections
}

\author{
Christopher Persichilli* for the ARIANNA Collaboration \\ Department of Physics and Astronomy, University of California Irvine \\ Irvine, CA 92697-4575, USA \\ E-mail: cpersicheuci.edu
}

The ARIANNA project seeks to observe cosmogenic neutrinos with energy in excess of $10^{16} \mathrm{eV}$ through the use of a grid of over 1000 independent radio detector stations. These stations search for the characteristic Askaryan radio pulses, from particle cascades generated in the ice by these neutrinos, with a bandwidth of $50-1000 \mathrm{MHz}$. Spaced a kilometer apart, this array would effectively survey approximately 750 cubic kilometers of Antarctic ice. Currently, the Hexagonal Radio Array (HRA) at the Moore's Bay site (Ross Ice Shelf) serves as a pilot for the ARIANNA concept. A neutrino signal analysis will be presented using data from two seasons of HRA operation. We will also discuss the projected sensitivity of the full ARIANNA array using real-world performance data from the HRA, and show that ARIANNA will be able to probe conservative models of the GZK neutrino flux.

35th International Cosmic Ray Conference

10-20 July, 2017

Bexco, Busan, Korea

${ }^{*}$ Speaker. 


\section{Introduction}

The detection of cosmogenic neutrinos has the potential to probe astrophysics which is beyond the reach of traditional cosmic ray astronomy. While protons with energy in excess of $10^{20} \mathrm{eV}$ interact with the $\mathrm{CMB}$ with an interaction length of about $50 \mathrm{Mpc}$, the range for similarly energetic neutrinos is greater than the size of the visible universe. In fact, the $\Delta$ resonance which limits charged CR propagation, (the GZK mechanism) $[1,2]$ is expected to produce a strong increase in the neutrino spectrum around $10^{18} \mathrm{eV}$ (the GZK flux) [3]. The expected flux at these energies, however, is still so low that the upper limits set by current experiments such as RICE, IceCube, Auger and ANITA [4] are still orders of magnitude above the most conservative, iron-only, flux models [5] for most energies (Figure 6).

The ARIANNA design aims to detect these ultra-high energy neutrinos by searching for characteristic Askaryan radio pulses generated by neutrino induced particle showers in ice [6]. Since the attenuation length for radio at these frequencies is on the order of $1 \mathrm{~km}$ in cold ice, which is abundant in Antarctica, a sparsely populated array can very economically survey much larger volumes than would be feasible with optical techniques. Additionally, ARIANNA's Moore's Bay site on the Ross Ice-Shelf benefits from the reflective interface between the bottom of the shelf and the sea below, allowing surface detectors to instrument the entire volume without drilling into the ice, and providing better sky coverage.

\section{Hardware Design and Performance}

Currently, ARIANNA consists of a seven station hexagonal array of neutrino detector stations (the HRA [7]) which was completed in December 2014, as well as a few purpose built cosmic ray stations with upward facing LPDA's. This submission will focus on the neutrino stations, but more information on the cosmic ray stations can be found in [8, 9]. In December 2015 all sites were upgraded to the latest DAq system, and a major revision of ARIANNA's battery systems was implemented. This has improved the live-time of the HRA, with each station having an average of 143 days of live-time during the 2016-2017 austral summer, with stations typically attaining 90\% livetime per day, see Figure 1.

The stations of the ARIANNA detector are autonomous and independent, each with their own self-contained solar power and communication systems. This allows the stations to operate throughout the austral summer while transmitting data to UCI for analysis in real time over WiFi, or Iridium satellite. Each station contains a four channel data acquisition (DAq) board which records a $128 \mathrm{~ns}$ waveform at $2 \mathrm{GSa} / \mathrm{s}$, described in detail in [10]. Each DAq channel is fed by a downward facing log-periodic dipole antenna (LPDA), which is directional and has an effective bandwidth of $50-1000 \mathrm{MHz}$ when buried in the snow. The antennas are placed in two co-polarized pairs, with a $6 \mathrm{~m}$ separation. In order to form a triggered event, a signal must pass a $4 \sigma$ high-and-low threshold (waveform crosses both thresholds within 10ns) above thermal $V_{R M S}$ on two of four channels within a $30 \mathrm{~ns}$ window, which leads to a typical thermal trigger rate measured in $\mathrm{mHz}$. The DAq system is also capable of performing an on-board veto to remove most events from narrow-band transmitters (see Section 4). 


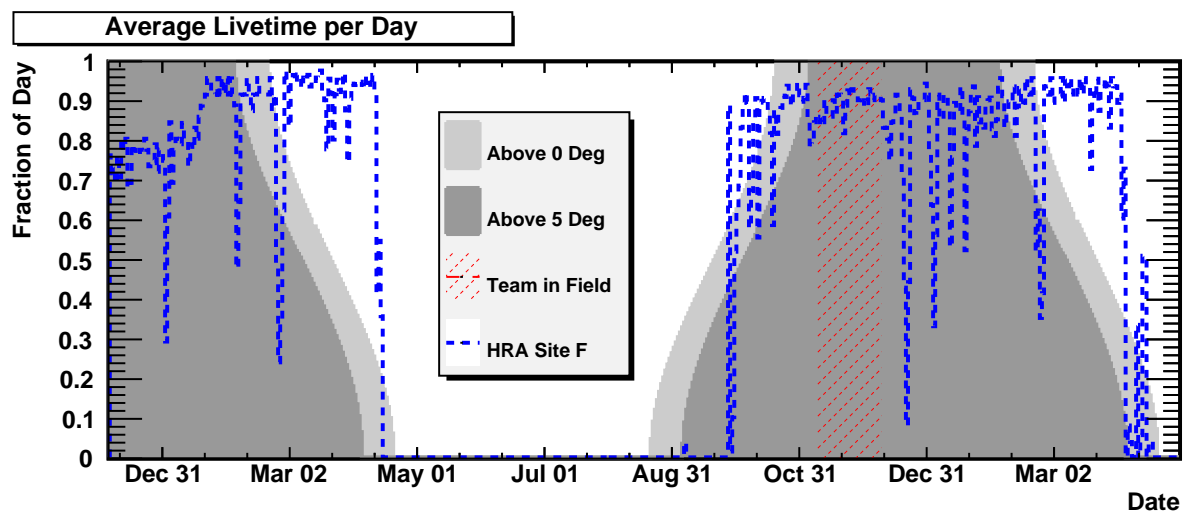

Figure 1: Livetime fraction per day for an HRA station, from December 2015 to April 2017. The grey shaded region shows the fraction of time the sun is above a certain elevation per day. The blue dashed line represents useful data taking time for analysis, adjusted for data transmission time and DAq deadtime due to event readout. Station operation configurations were optimized for livetime in mid January 2016. Large dips are due to a combination of bulk data transfers and some particularly stormy periods. Stations typically show livetimes near $90 \%$.

\section{The Neutrino Signal Space}

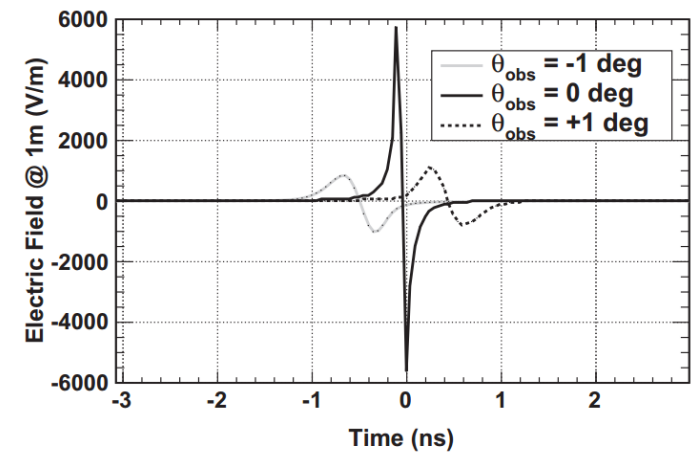

(a)

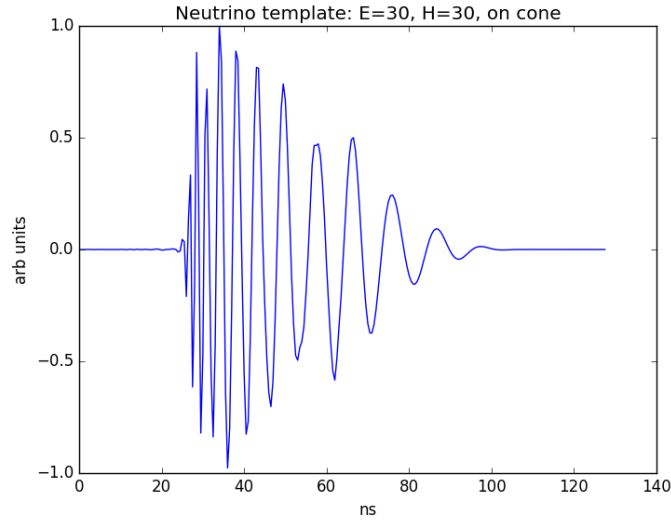

(b)

Figure 2: (a) Simulated raw E-field due to a neutrino induced particle shower in ice. (b) A neutrino signal template after convolving (a) with the amplifier and antenna response. The fast, bipolar pulse generated by the Askaryan effect leads to a characteristic waveform with a chirp-like time structure due to the dispersion of the antenna and amplifiers. See [11]

The primary technique for separating the neutrino signal from background consists of a template matching procedure. Templates for the neutrino signal generated by convolving a ZHS derived Askaryan pulse with the measured response of our antennas and amplifiers [11]. For each event we can define a parameter, $\chi$, to be the maximum correlation coefficient of any DAq channel to a reference template, which we have chosen to be $\mathrm{E}$ and $\mathrm{H}$ angles of $30^{\circ}$, see Figure $2 \mathrm{~b}$. 
This allows us to perform an analysis which is independent of the directional reconstruction. This parameter is an excellent discriminator against thermal noise, which comprises the bulk of our triggered events (Figure 3). A similar template matching procedure has also been validated by successfully identifying cosmic rays in the upward facing antennas of ARIANNA's cosmic ray stations $[12,8]$.

CDF's of Min-Bias Data vs Neutrino Signal
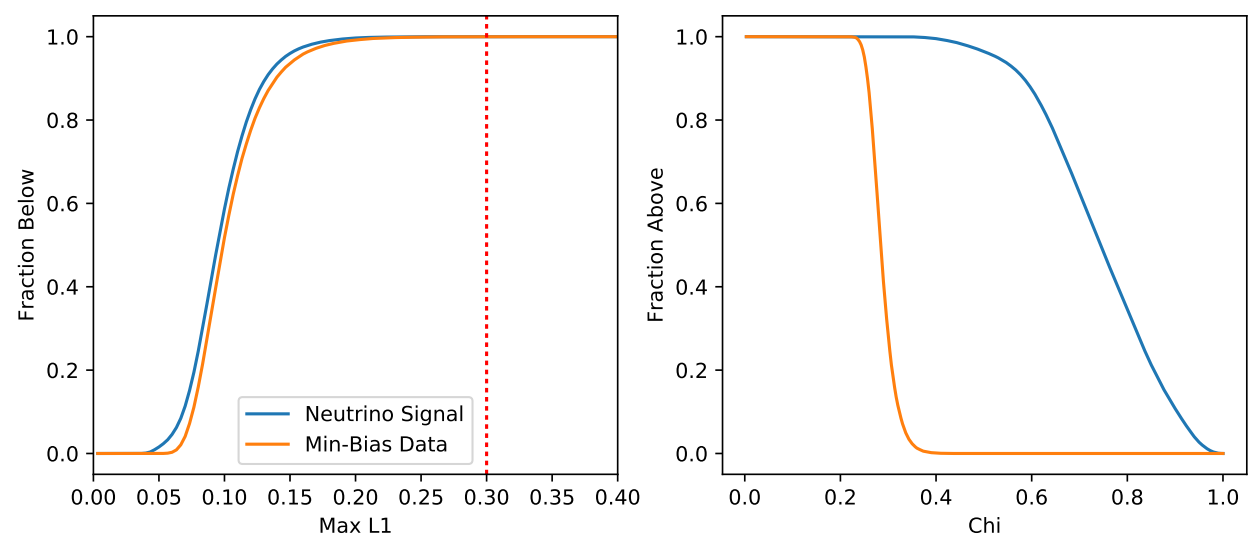

Figure 3: Distributions of the $\chi$ and $L 1$ variables for the neutrino signal space and min-bias data from the HRA. This shows that $\chi$ discriminates very efficiently against thermal noise. The onboard veto of $L 1>0.3$ is efficient at vetoing narrow band transmitters, while only rejecting 1.8 in $10^{5}$ simulated neutrino signals.

These same templates are used in combination with our ShelfMC Monte-Carlo [13] in order to create the neutrino signal space for this analysis. ShelfMC generates neutrino interaction vertices and calculates a signal strength and arrival direction at a simulated ARIANNA detector, accounting for ice propagation, and generates triggers based on the same $4 \sigma$ threshold used in our current stations. The amplitude and directional distributions are combined with the neutrino templates and random noise in order to generate simulated neutrino events for analysis.

\section{Sources of Noise and Background}

ARIANNA's Moore's Bay site is remarkably radio quiet, but there still exist some sources of background which must be considered. The bulk of triggered events are random in nature, either generated by thermal noise in our amplifiers, or from galactic radio emission. This type of background is easily discriminated against by the $\chi$ parameter, and trigger rates are managed by requiring multi-channel trigger coincidence.

The stations also record occasional short bursts of narrow-band anthropogenic noise from air traffic control, and similar sources. Since the neutrino signal is broad-band in nature, a simple cut against highly peaked frequency spectra is effective at removing this background. We define a parameter as part of our station level 1 trigger $(L 1)$ which is the ratio of the power in the highest power FFT bin to the total power in all other bins. We find that a cut on events with $L 1>0.3$ is sufficient to remove our narrow-band background, with a simulated neutrino signal rejection rate of $1.8 \times 10^{-5}$ (Figure 3). 
Another source of noise are events which are associated with periods of storm and high winds at the ARIANNA site. These stormy periods generate bursts of high events rates, which are somewhat pulsed in nature, and match neutrino templates more closely than thermal noise. The exact mechanism behind this RF noise is not yet understood, however, we will show that they can be efficiently vetoed based on their waveform properties.

The primary background of concern for ARIANNA comes from atmospheric cosmic ray showers. These showers generate radio pulses in a similar process to those initiated by neutrinos in the ice, and as such, generate similar waveforms in the ARIANNA detector. Future designs for ARIANNA account for this by including upward facing antennas. In this way, upward going signals (neutrinos) can be easily distinguished from downward going signals (cosmic rays) due to the directional gain of the LPDA's. These upward facing antennas are not yet incorporated in the HRA stations, however, so cosmic rays remain a significant background.

\section{Analysis of Triggered Events}
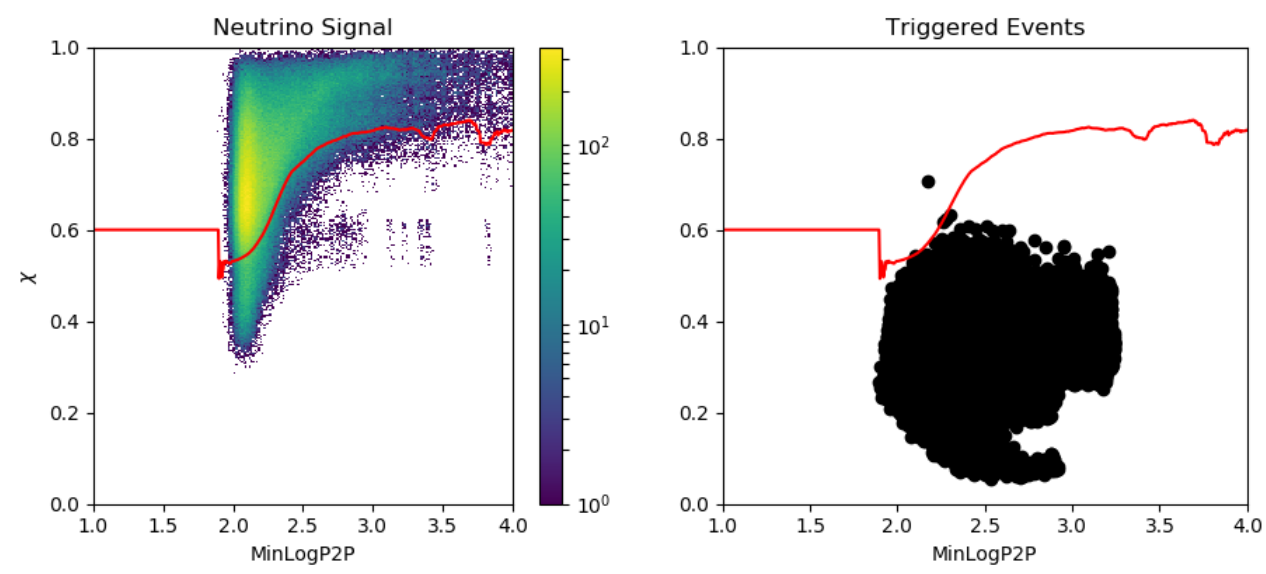

Figure 4: Distribution on the signal space and all thermal triggers in $\chi$ vs MinLogP2P (see Section 5). The red line represents the $90 \%$ efficiency cut for a sliding 0.1 decade bin in MinLogP2P. The significance of outlying triggered event (Fig 5) is discussed in Section 5.

Looking at the distribution of the neutrino signal in $\chi$ vs amplitude (figure 4), there is a clear trend towards higher $\chi$ values with increasing amplitude, due to the improved signal-to-noise ratio. We would expect that as our background shows a greater signal to noise ratio, it should also be more easily distinguishable from the neutrino signal. This allows for a more efficient cut on the data then simply setting a fixed value of $\chi$. Here we have set a $90 \%$ efficient cut on a 0.1 decade sliding bin in MinLogP2P, which is $\log _{10}\left(\frac{V_{p t p}}{m V}\right)$ where $V_{p t p}$ is the peak-to-peak voltage of the minimum amplitude channel. The resulting line will define our neutrino signal space.

Data from all HRA stations was analyzed from the last major hardware revision in December 2015, until this season's winter shutdown around April $15^{\text {th }}$, 2017. Of all triggered events in this time period, exactly one event survives the cuts mentioned in this paper, see Figures 4 and 5. This event was not associated with a cluster of high-rate wind related events, nor were there any coincident events in any upward-facing antennas of the cosmic ray stations. This lack of a coincidence 
is not unexpected however, due to the asynchronous nature of the stations, and the fact that only certain cosmic ray footprints have the possibility of triggering multiple stations. Unfortunately, this leaves us unable to verify whether this event was, in fact, due to a cosmic ray (as opposed to an exceedingly (un)lucky neutrino observation). Of course, this is a problem that was anticipated, and is the justification for the inclusion of upward facing LPDA's, which can clearly discriminate the directionality of the signal, in future ARIANNA designs.
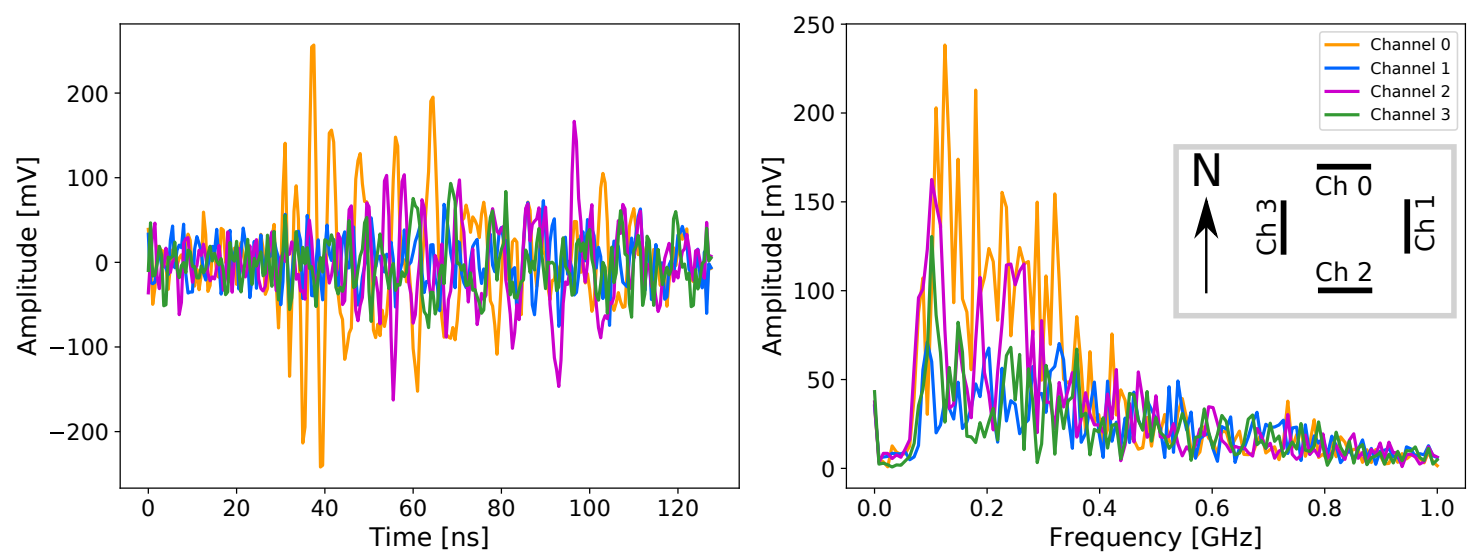

Figure 5: Waveform and FFT of the outlying event in Figure 4. This event has a correlation value of $\chi=0.71$, and was recorded at ARIANNA Site A at 10:25:03 UTC on March 27 $7^{\text {th }}, 2017$. The inset shows the positions and polarizations of the LPDA's for each DAq channel.

\section{Flux Limits}

In the absence of observed events, and assuming that the upward facing antennas in future ARIANNA stations can tag cosmic rays such that there are no background events, we can use the Neyman formalism to place a $90 \%$ confidence upper limit of 2.3 events in a given energy bin. This results in the expression

$$
E^{2} \Phi(E) \leq \frac{2.3 E L(E)}{\ln 10 d \log E \varepsilon V_{\text {eff }} \Omega t_{\text {live }}}
$$

for the flux limit, where $V_{e f f} \Omega$ is the effective volume averaged over all flavors, $L(E)$ is the water equivalent interaction length calculated using the cross section in [14], $\varepsilon$ is the analysis efficiency of the neutrino signal, and $t_{\text {live }}$ is the total livetime of the detector. Using an analysis efficiency of $90 \%$, the single station $V_{\text {eff }} \Omega$ from the ShelfMC Monte-Carlo times 1296 stations, and a livetime extrapolated from the average livetime over the 2016-2017 season, we can make a 5 year flux limit projection for the full ARIANNA array, see Figure 6.

\section{Conclusions and Discussion}

We have demonstrated that the ARIANNA hardware is capable of operating with a near 90\% livetime efficiency during the austral summer. The extremely radio-quiet environment at 


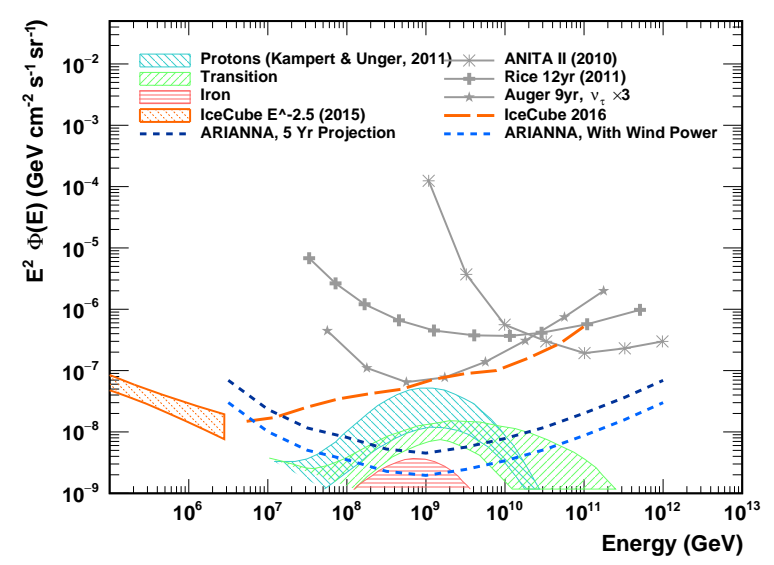

Figure 6: Projected flux limits for 5 years of operation of the full 1296 station ARIANNA array at the Moore's Bay site. Using the analysis efficiency and actual livetime fraction from the 20162017 HRA data, we project the capability to probe a number of conservative flux models. The addition of wind power at ARIANNA (currently under development) has the potential to double our total livetime. IceCube limits and spectra from $[4,15]$. RICE, Auger, and ANITA limits from $[16,17,18]$. Flux models from [5]. For details of the calculation see section 6.

the Moore's bay site allows for a rejection of most noise sources with a 90\% signal efficiency, and while cosmic rays remain a significant background in the pilot design, this will be mitigated by the inclusion of upward facing antennas in future stations. After assuming that cosmic rays will be efficiently tagged in the future, it is possible to make a projection for a full ARIANNA array using the efficiency of the current analysis. Even assuming that there are no future improvements to the livetime fraction of the stations, a 5 year deployment would be able to probe transition models of the GZK flux around $10^{18} \mathrm{eV}$, Figure 6. With some additional optimization, such as the addition of reliable wind power, and vertically polarized dipole antennas, we can extend the sensitivity of ARIANNA down to conservative, proton only, models. See [19] for discussion of possible optimizations of the ARIANNA array.

\section{Acknowledgements}

We wish to thank the US National Science Foundation for its support of the ARIANNA project under grant PHY-1607719. We also acknowledge support from National Research Nuclear University MEPhI (Moscow Engineering Physics Institute), and from the German Research Foundation (DFG, grant NE 2031/1-1). We also wish to thank the Antarctic Support Contractor and the McMurdo Station staff for their outstanding cooperation and support in field planning and operations.

\section{References}

[1] K. Greisen, End to the cosmic-ray spectrum?, Physical Review Letters 16 (apr, 1966) 748-750.

[2] G. Zatsepin and V. Kuz'min, Upper limit of the spectrum of cosmic rays, JETP Letters 4 (1966) 78.

[3] F. W. Stecker, Ultrahigh Energy Photons, Electrons, and Neutrinos, the Microwave Background, and the Universal Cosmic-Ray Hypothesis, Astrophysics and Space Science 20 (Jan., 1973) 47-57. 
[4] IceCube Collaboration, M. Aartsen et al., Constraints on ultrahigh-energy cosmic-ray sources from a search for neutrinos above $10 \mathrm{PeV}$ with IceCube, Physical Review Letters 117 (dec, 2016).

[5] K.-H. Kampert and M. Unger, Measurements of the cosmic ray composition with air shower experiments, Astroparticle Physics 35 (may, 2012) 660-678.

[6] ANITA Collaboration, P. W. Gorham et al., Observations of the askaryan effect in ice, Physical Review Letters 99 (oct, 2007).

[7] ARIANNA Collaboration, S. W. Barwick et al., A first search for cosmogenic neutrinos with the ARIANNA Hexagonal Radio Array, Astroparticle Physics 70 (Oct., 2015) 12-26.

[8] A. Nelles for the ARIANNA Collaboration, Cosmic-ray capabilities of the ARIANNA neutrino experiment, These Proceedings (2017). PoS(ICRC2017)399.

[9] S. Wang et al., for the ARIANNA Collaboration, Calibration, Performance, and Cosmic Ray Detection of ARIANNA-HCR Prototype Station, These Proceedings (2017). PoS(ICRC2017)358.

[10] ARIANNA Collaboration, S. W. Barwick et al., Design and Performance of the ARIANNA Hexagonal Radio Array Systems, IEEE Transactions on Nuclear Science (2015) [arXiv: 1410 . 7369].

[11] ARIANNA Collaboration, S. W. Barwick et al., Time-domain response of the ARIANNA detector, Astroparticle Physics 62 (Mar., 2015) 139-151, [arXiv: 1406.0820 ].

[12] ARIANNA Collaboration, S. Barwick et al., Radio detection of air showers with the ARIANNA experiment on the ross ice shelf, Astroparticle Physics 90 (apr, 2017) 50-68.

[13] K. Dookayka, Characterizing the Search for Ultra-High Energy Neutrinos with the ARIANNA Detector. PhD thesis, University of California, Irvine, 2011.

[14] A. Connolly et al., Calculation of high energy neutrino-nucleon cross sections and uncertainties using the martin-stirling-thorne-watt parton distribution functions and implications for future experiments, Physical Review D $\mathbf{8 3}$ (jun, 2011).

[15] IceCube Collaboration, M. Aartsen et al., Atmospheric and astrophysical neutrinos above $1 \mathrm{TeV}$ interacting in IceCube, Physical Review D 91 (jan, 2015).

[16] RICE Collaboration, I. Kravchenko et al., Updated results from the RICE experiment and future prospects for ultra-high energy neutrino detection at the south pole, Physical Review D $\mathbf{8 5}$ (mar, 2012).

[17] Pierre Auger Observatory Collaboration, P. Abreu et al., Search for point-like sources of ultrahigh energy neutrinos at the pierre auger observatory and improved limit on the diffuse flux of tau neutrinos, The Astrophysical Journal 755 (jul, 2012) L4.

[18] ANITA Collaboration, P. W. Gorham et al., Observational constraints on the ultrahigh energy cosmic neutrino flux from the second flight of the ANITA experiment, Physical Review D 82 (jul, 2010).

[19] S. Barwick for the ARIANNA Collaboration, Optimization of ARIANNA station configuration, These Proceedings (2017). PoS(ICRC2017)1042. 